\title{
FAVOURABLE TUMOURS OF THE POSTERIOR CRANIAL FOSSA
}

\author{
Leslie Olivfr, M.B., F.R.C.S., F.A.C.S. \\ Neurosurgeon, Charing Cross Hospital, West London Hospital, Royal Northern Hospital, West End Hospital for \\ Neurology and Neurosurgery, London
}

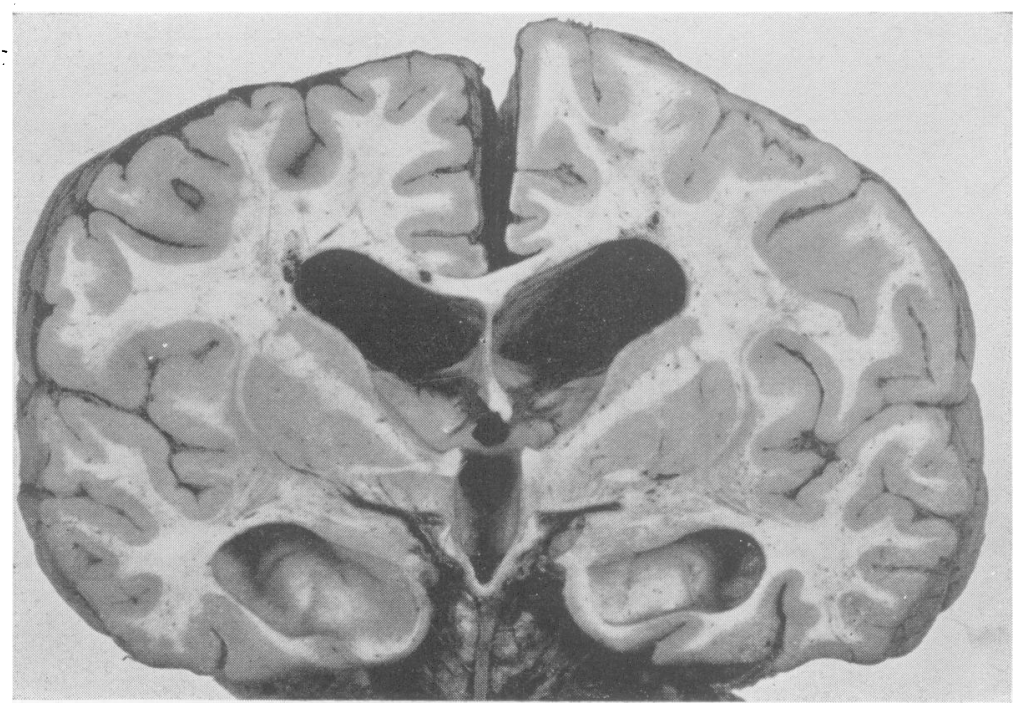

FIG. I.-Enlargement of the lateral and third ventricles produced by a tumour in the posterior fossa.

If the diagnosis of a benign intracranial tumour is made soon enough, a favourable outcome can nowadays be expected after operation. Therefore, when a patient is shown to have an intracranial tumour, its pathological nature must be proven beyond doubt. Benign tumours may occur anywhere inside the skull, but in this article discussion is limited to those of the posterior fossa.

One or more of the following effects may be brought about by expanding lesions of the posterior fossa. The flow of cerebrospinal fluid through the aqueduct and fourth ventricle may be obstructed and the resulting hydrocephalus is revealed by frontal headache, vomiting and papilloedema (Fig. I). Suboccipital headache occurs in some cases in association with descent of a ccrebcllar pressure cone through the foramen magnum. A lesion expanding from a lateral position towards the midline may cause lateral nystagmus, which is slower and of greater amplitude when the eyes look towards the side of the lesion. Hypotonia, incoordination, intention tremor, dysdiadochokinesia and past-pointing may be demonstrated in the arm on the side of the tumour and the outstrctched arm may deviate spontaneously outwards and downwards. Sometimes there is hypotonia and incoordination of the homolateral leg and veering or falling towards the side of the lesion. A tumour expanding in the midline behind the brain-stem tends to cause ataxia and hypotonia of the lower limbs.

Pressure may occur on pyramidal fibres on aither or both sides. Contralateral pyramidal signs are produced by direct pressure of the tumour on the pons, whereas homolateral pyramidal signs are produced by the cerebral peduncle of the oppositc side being forced against the free edge of the tentorium cerebelli and consequent pressure on pyramidal fibres which cross below in the medulla oblongata to the side of the lesion. Extrinsic 


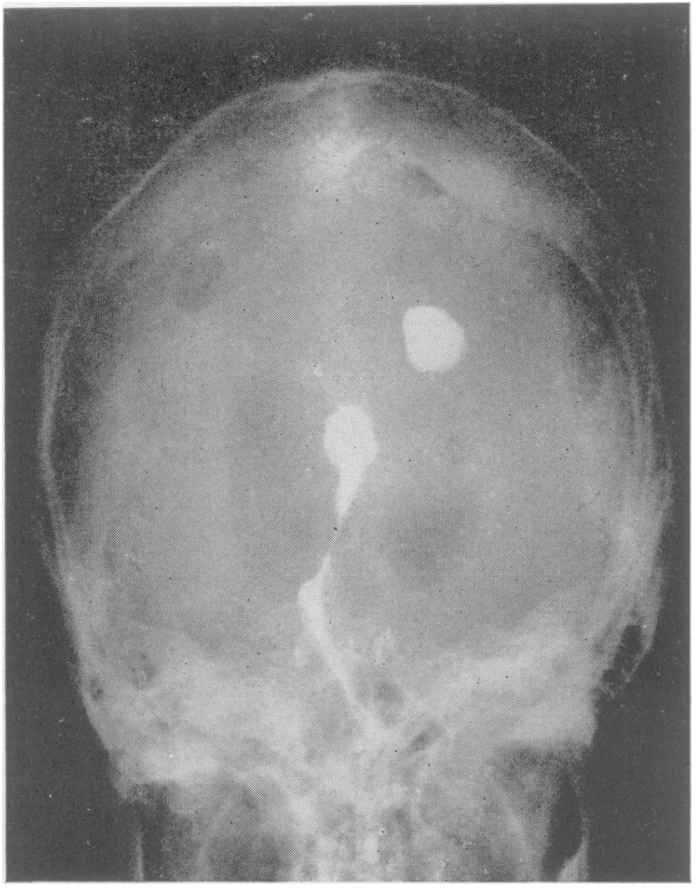

FIG. 2.- Ventriculography with air (negative contrast) and Myodil (positive contrast). The air is in the anterior horns of the lateral ventricles and anterior end of the third ventricle showing advanced hydrocephalus. On the right there is some Myodil in the occipital horn. The rest outlines the posterior end of the distended third ventricle, the aqueduct displaced to the left with its upper part distended and its lower part compressed, and the fourth ventricle which is deformed and displaced to the left. These effects were produced by an acoustic tumour on the right.

tumours are likely to compress the cranial nerves of the posterior fossa early in their evolution, whereas intrinsic tumours tend to compress them late, or not at all. For reasons as yet unexplained, cranial nerve palsies occasionally occur on the side opposite to the tumour. Severe compression of the medulla oblongata may occur from an expanding lesion arising anywhere in the posterior fossa, and may give rise to vertigo, dysarthria, dysphagia, repeated vomiting, bradycardia, slowing of respiration and eventually Cheyne-Stokes respiration.

Unilateral or bilateral external rectus paralysis occurs with some tumours in the posterior fossa due to stretching of the abducens nerves. This is brought about by descent of the brain-stem produced by increasing pressure in the ventricles above the tentorium.

Bilateral inferior temporal scotomas or bitemporal lower quadrantic field defects (never a complete bitemporal hemianopia) may result from

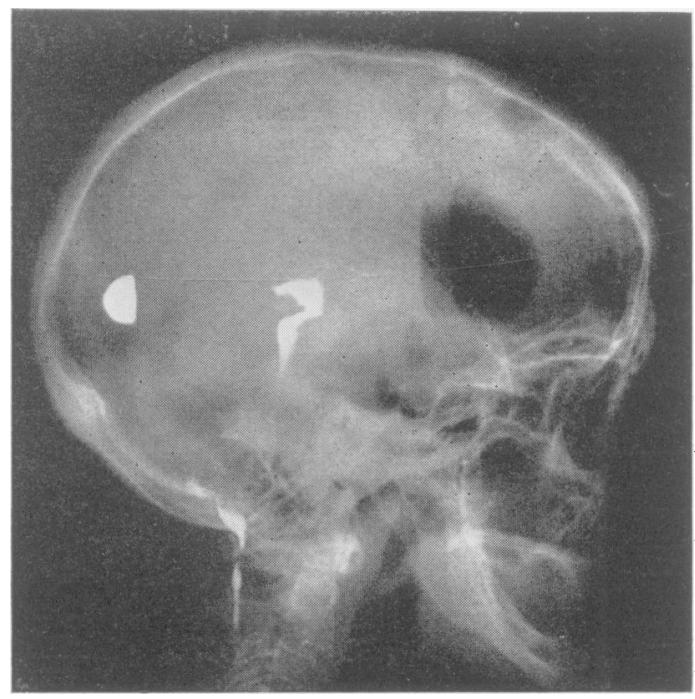

Fig. 3.-A lateral view taken in the same case as Fig. 2. Air shows distension of the lateral ventricles, and Myodil shows sharp forward angulation of the aqueduct which is also distended above and constricted below.

pressure on the optic chiasm by the anterior.end of the distended third ventricle.

Slowly expanding intrinsic cerebellar tumours (e.g. astrocytomas and haemangioblastomas) quite often cause little or no cerebellar disturbance and give no evidence of involvement of cranial nerves or the brain-stem, but present with manifestations of raised intracranial pressure alone. Such tumours are easily mistaken for ' silent' tumours arising in other parts of the cranium (e.g. prefrontal and pretemporal regions). Furthermore, frontal and even parietal lesions occasionally produce mild contralateral ' cerebellar' signs. Rapidly expanding lesions, such as cystic tumours and cerebellar haemorrhage, cause acute medullary compression and acute hydrocephalus.

\section{Investigations}

Ventriculography usually reveals dilatation of the lateral ventricles and third ventricle. Displacement, deformity and dilatation of the aqueduct and fourth ventricle may also be demonstrated. Tumours in the posterior fossa sometimes cause upward displacement of the third ventricle, or upward curving of the posterior horn of the lateral ventricle on the side of a laterally placed tumour. Marked indentation of the occipital horn occurs with dumb-bell meningiomas arising from the tentorium and growing supraand infra-tentorially. Positive contrast ventriculography with Myodil (I to $2 \mathrm{ml}$.) injected through the anterior horn of one of the lateral 
ventricles gives more reliable information regarding the aqueduct and fourth ventricle than air ventriculography (Figs. 2 and 3 ). However strong the clinical evidence of a posterior fossa tumour may be, ventriculography should always be done except, perhaps, when there is indisputable evidence of an acoustic tumour, which often produces a characteristic syndrome. Vertebral angiography is more difficult and more dangerous than carotid angiography. Therefore it is not employed if the necessary information can be obtained otherwise. Aneurysms, vascular malformations and vascular tumours are outlined in angiograms.

\section{Types of Benign Tumours}

Astrocy'tomas are the commonest intrinsic cerebellar tumours. The maximum incidence is during the first two decades. In the cerebellum most astrocytomas are cystic and benign (Grade I). Frequently there is a large cyst with a small mural tumour. The cystic fluid is yellow and contains a large amount of protein. Removal of the mural nodule is usually all that is required.

Haemangioblastomas (Syn. Angioreticulomas). These tumours occur most often in the cerebellum; they are rare elsewhere in the nervous system. They are more often cystic than solid. The cysts tend to be large and contain mural tumours like those in cystic astrocytomas of the cerebellum. Unlike the latter tumours however, haemangioblastomas are found most often in adults, and they show themselves earlier in women than in men. Thus in women the maximum incidence occurs between twenty and forty years, whereas in men it is between forty and sixty years. Sometimes these tumours are familial; they then tend to be multiple. Therefore vertebral angiography should be done for suspected haemangioblastomas when there is a family history of these tumours, otherwise multiple tumours may be overlooked at operation. The association with haemangioblastomas elsewhere and cysts in the liver, pancreas or kidney, is known as Lindau's syndrome. Angioma of the retina (von Hippel's disease) may be one of the lesions forming part of Lindau's syndrome.

Acoustic Nerve Tumours (Syn. Acoustic Neurinomas, Schwannomas, Perineural Fibroblastomas or Neurilemmomas). These tumours are thought to arise from the endoneurium of the acoustic nerve within the internal auditory meatus. They grow in the subdural space, and as they expand come into relationship with the trigeminal nerve, cerebellum, pons, and eventually also with the ninth, tenth, eleventh and twelfth cranial nerves (Fig. 4). They are usually solid tumours but are commonly surrounded by a cyst formed by the arach-

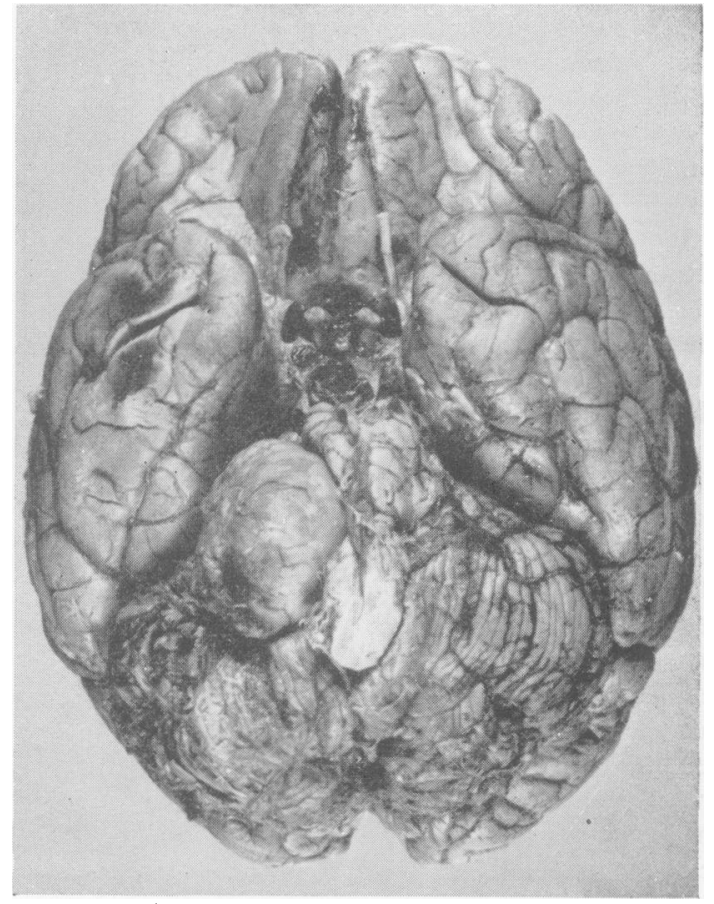

Fig. 4.-A right-sided (readers' left) acoustic tumour. There is a severe degree of indentation of the pons and medulla oblongata.

noid. Bilateral acoustic tumours are sometimes found in the 'central' type of von Recklinghausen's neurofibromatosis. Acoustic tumours are composed of interlacing bundles of spindle cells containing elongated nuclei. The cells tend to be arranged in rows ('palisading') and some form whorls around blood vessels. Reticulum fibrils are conspicuous between cells. Nerve fibres are rarely seen.

These tumours affect both sexes equally, and symptoms begin in middle age and have usually been present for two to three years before the neurosurgeon is consulted. The first symptom is gradually progressive deafness in one ear, frequently accompanied by tinnitus and dizziness (severe vertigo is rare). Involvement of the vestibular division of the eighth cranial nerve is demonstrated by the caloric test.

Paraesthesia in the distribution of the fifth cranial nerve is common, but trigeminal pain is rare. Absence of the corneal reflex may be the only sign of involvement of this nerve. Strangely enough, there is little or no evidence of compression of the facial nerve. There may be aching pain in the occipital muscles and tenderness behind the homolateral mastoid process. Later there are cerebellar signs. In some cases, compression of the brain-stem produces pyramidal signs on either 
or both sides. Later there are signs of increased intracranial pressure (headache, vomiting and papilloedema, abducens nerve paralysis). Extension of the tumour to the tenth and eleventh cranial nerves causes unilateral palatal, pharyngeal and laryngeal paralysis and also partial paralysis of the sternomastoid and trapezius muscles.

Radiography often shows enlargement of the internal auditory meatus or sometimes considerable erosion of the petrous bone. When the syndrome is atypical, positive contrast ventriculography (Myodil I to $2 \mathrm{ml}$.) is carried out to show the aqueduct and fourth ventricle.

Removal of acoustic tumours is carried out through a unilateral approach with the patient in the sitting position. Operation still has a considerable mortality (up to 25 per cent.) because these tumours are usually referred to the neurosurgeon when there is marked indentation of the brain-stem and advanced hydrocephalus. Decompression alone is valueless, for acoustic tumours are firmly attached to the petrous bone and therefore the pressure on the brain-stem is not relieved. The facial nerve is not seen during operation, and facial palsy usually results. It is treated by facialhypoglossal or facial-accessory anastomosis.

Meningiomas in the posterior fossa may be attached to any part of the dura mater, but usually arise somewhere along the transverse or sigmoid sinuses. Sometimes they grow in the cerebellopontine angle and mimic acoustic tumours. Dumb-bell meningiomas of the tentorium are rar $\mathrm{e}$ tumours which grow upward to indent the parieto-occipital lobe and downward into the posterior fossa. In the removal of meningiomas of the posterior fossa, the transverse sinus on one side may be divided or excised when necessary, as there is usually an anastomotic channel between the two transverse sinuses. The presence of this connection may, however, be verified preoperatively by venography (the superior longitudinal sinus is injected through a burr-hole with $20 \mathrm{ml}$. of 42.5 per cent. diodone solution) and serial $\mathrm{X}$-rays are taken.

Epidermoid Tumours (Syn. Cholesteatomas, Pearly Tumours of Cruveilhier). These are congenital tumours arising from the meninges and consisting of a capsule of epidermal tissue enclosing a mass of epithelial debris and cholesterol crystals. They are not to be confused with the inflammatory type of ' cholesteatoma' caused by chronic infection of the middle ear and mastoid. The whole of the contents of the 'cyst' and as much of the capsule as possible must be removed, for if some cholesterol remains it causes an aseptic meningitis which can be fatal.

\section{NEWCASTLE SYMPOSIUM}

(Postgraduate Medical Journal, April 1960)

Price 6s. 6d. post free

THE THROMBOGENIC HYPOTHESIS AND ITS IMPLICATIONS

J. B. Duguid, M.D.

THE MANAGEMENT OF ACUTE RENAL FAILURE

Walter Elliott, M.B., B.S., Molly Hill, M.B., Ch.B., M.R.C.P., D.R.C.O.G., David N. S. Kerr, M.Sc., M.R.C.P., M.R.C.P.(Edin.), and Rosalind Ashcroft, Ph.D.

CURRENT RESEARCH IN MULTIPLE SCLEROSIS

E. J. Field, M.D., M.S., Ph.D., and Henry Miller, M.D., F.R.C.P.

THE SURGICAL' TREATMENT OF PARKINSON'S DISEASE

John Hankinson, M.B.(Lond.), F.R.C.S.

CHRONIC OTITIS MEDIA

F. McGuckin, M.D., F.R.C.S.
SOME RECENT TRENDS AND DEVELOPMENTS IN DERMATOLOGY

G. Holti, M.D., and John T. Ingram, M.D., F.R.C.P.

SOCIAL AND ENVIRONMENTAL FACTORS IN CHRONIC BRONCHITIS IN NEWCASTLE UPON TYNE

A. G. Ogilvie, M.D., F.R.C.P.

ENQUIRIES INTO MENTAL DISORDER IN OLD AGE

Martin Roth, M.D., F.R.C.P., and D. W. K. Kay, M.A., B.M., D.P.M.

STUDIES OF LUNG FUNCTION IN CHILDREN

L. B. Strang, M.B., M.R.C.P., and S. D. M. Court, M.D., F.R.C.P., D.C.H.

EMBOLECTOMY IN THE LIMB ARTERIES

David J. Tibbs, M.C., M.S.(Lond.), F.R.C.S. 